\title{
Acute Toxoplasmosis Mimicking Melanoma Metastases: Review of Conditions Causing False-Positive Results on ${ }^{18}$ F-FDG PET/CT
}

\author{
K. Ivanova ${ }^{a} \quad$ K. Glatz ${ }^{b}$ A. Zippelius ${ }^{c}$ G. Nicolas ${ }^{d}$ P. Itin ${ }^{a}$ \\ Departments of a Dermatology, ${ }^{b}$ Pathology, ${ }^{c}$ Medical Oncology and ${ }^{d}$ Nuclear Medicine, University Hospital Basel, \\ Basel, Switzerland
}

\section{Key Words}

Toxoplasmosis · Malignant melanoma . Fluorine-18-fluorodeoxyglucose positron emission tomography-computed tomography

\begin{abstract}
Invasive malignant melanoma is the most common fatal form of skin cancer. Fluorine18-fluorodeoxyglucose positron emission tomography-computed tomography demonstrates a very high sensitivity and specificity for the detection of melanoma metastases. Here, we report an unusual case of toxoplasma lymphadenitis in a male adult patient mimicking a malignant cervical lymphadenopathy. Toxoplasmosis is a zoonosis caused by the intracellular parasite Toxoplasma gondii, which is usually asymptomatic in immunocompetent hosts.
\end{abstract}

Copyright $\odot 2013$ S. Karger AG, Basel

\section{Case Report}

In July 2011, a 46-year-old male patient complained about a tender nodule on the jaw. At this time the patient felt tired and weak, and he reported a weight loss of $7 \mathrm{~kg}$ during the last 6 months. At that time, he did not take any medication and did not show any signs of infection or other systemic symptoms such as fever or night sweats. His medical history was remarkable for malignant melanoma on the left ear in May 2009, with tumor thickness Breslow $0.98 \mathrm{~mm}$, Clark level IV, which had been treated with local surgical excision margins of $1 \mathrm{~cm}$. Sentinel lymph node biopsy and PET/CT did not show any signs of metastatic disease (fig. 1A). The staging workup revealed pT1aNOM0, disease stage IA according to the American Joint Committee on Cancer 2009. The patient's personal history was positive for ligament rupture on the right leg in 1982. The patient owns a pet cat.

On physical examination, we found a tender $2-\mathrm{cm}$ enlarged submental lymph node on the left side in the draining area of the primary melanoma. The melanoma scar, skin, mucous membranes and genital area appeared normal. Laboratory findings including a complete blood cell count with leukocyte differential count, chemistry panel, inclusive CRP, creatine kinase, liver enzymes, HIV screening and S-100 were normal. Cervical sonography showed two neighboring, inhomogeneous, hypoechogenic and hyperperfused submental lymph nodes with a size of $2.6 \times 0.9 \times 1.1 \mathrm{~cm}$ on the left side, which had a round shape and showed absent fat hilum. They were suspicious for malignancy. Another lymph node, on the right side, with size of $0.3 \mathrm{~cm}$ appeared abnormal because it was hypoechogenic and hyperperfused. In our case, the result of the fine-needle aspiration (FNA) was nonspecific. It consisted of predominantly small cleaved lymphocytes, occasional noncleaved cells, very few tingible body macrophages, and slightly atypical histiocytes as in reactive lymph node hyperplasia. On PET/CT disseminated lymphadenopathy (submental, cervical, axillar, intrahilar, mediastinal, mesenteric, suprainguinal, inguinal) and multiple lung nodules have been documented (fig. 1B). Based on these findings we suspected metastatic melanoma and performed a lymphadenectomy. However, the histopathologic examination of both submental lymph nodes revealed the classic signs of the so-called PiringerKuchinka disease. It is characterized by the presence of epithelioid cell granulomas, monocytoid cells, sinus histiocytosis and numerous tingible body macrophages in the germinal centers (fig. 2). These histological findings are highly specific for toxoplasmosis. The serological tests demonstrated high positive IgM and IgG antibodies with low IgG avidity, a typical constellation for an acute infection with Toxoplasma gondii. On this clinicopathological and serological basis, we confirmed the diagnosis of acute toxoplasmosis. We did not treat the infection, because our patient was immunocompetent. To be sure that none of the lymph nodes persist and the infection resolves, PET/CT has been repeated 3 months later and the disseminated lymphadenopathy

\section{KARGER}

E-Mail karger@karger.com

www.karger.com/drm
(C) 2013 S. Karger AG, Base

$1018-8665 / 12 / 2254-0349 \$ 38.00 / 0$
Dr. Katja Ivanova

Department of Dermatology, University Hospital Basel Petersgraben 4

$\mathrm{CH}-4031$ Basel (Switzerland)

E-Mailivanovak@uhbs.ch 


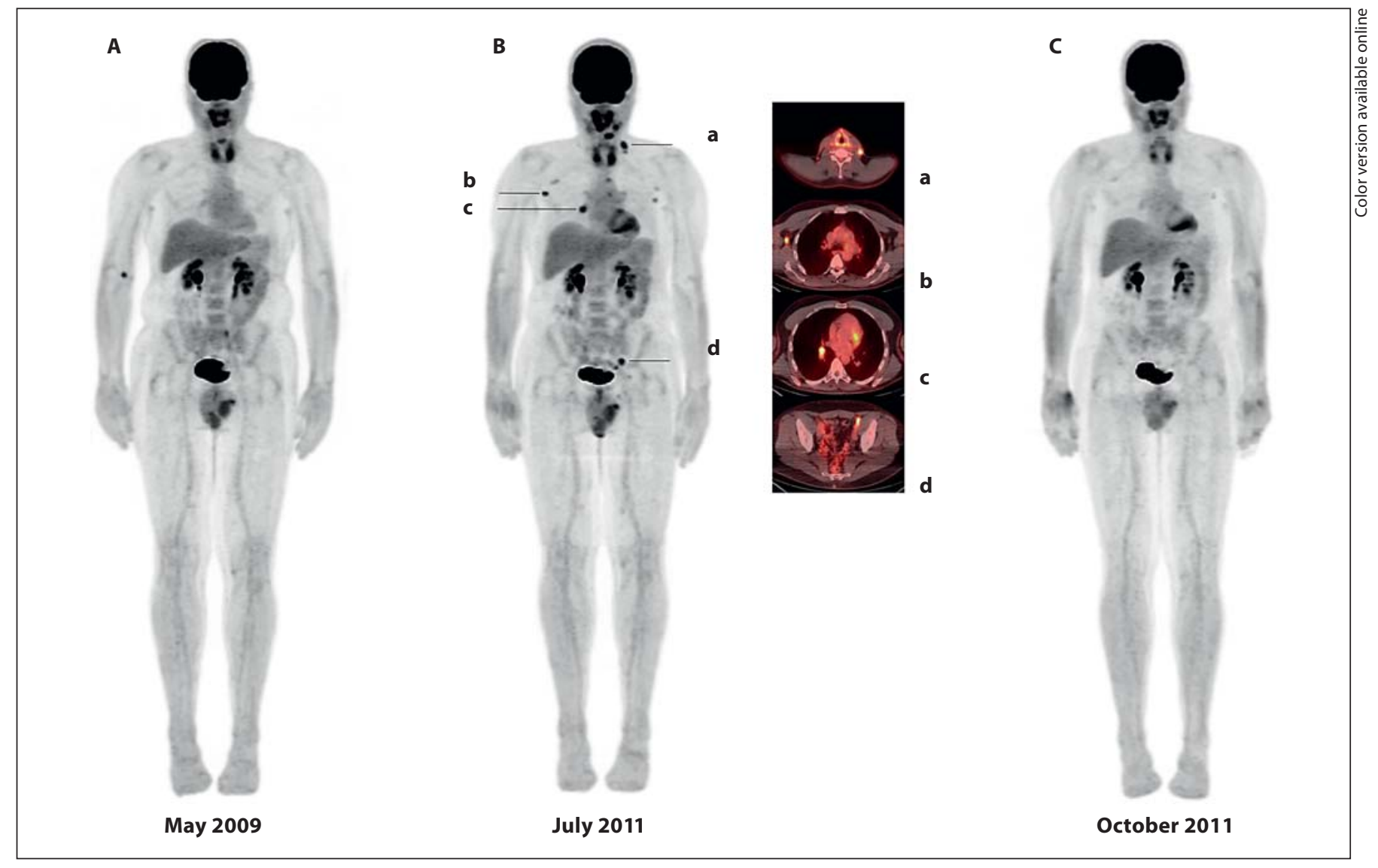

Fig. 1. A Maximum intensity projection of the initial whole-body ${ }^{18}$ F-FDG PET/CT showing no suspicious foci of uptake. B Whole body restaging showing several foci of uptake. The transaxial fusion images show area of increased uptake in the left cervical (a), right axillary (b), right hilary (c) and left external iliacal (d) regions consistent with metastatic lymphadenopathy. C Spontaneous normalization of the glucose metabolism in the previously described area of increased uptake.
Fig. 2. Characteristic findings of toxoplasma lymphadenitis. Lymph node capsule with chronic inflammation on the left. Large hyperplastic follicle center with numerous tingible body macrophages (short arrow). Cluster of parafollicular B lymphocytes with abundant pale staining cytoplasm resembling monocytes (long arrow). Small epitheloid cell granuloma (frame). HE. $\times 100$.

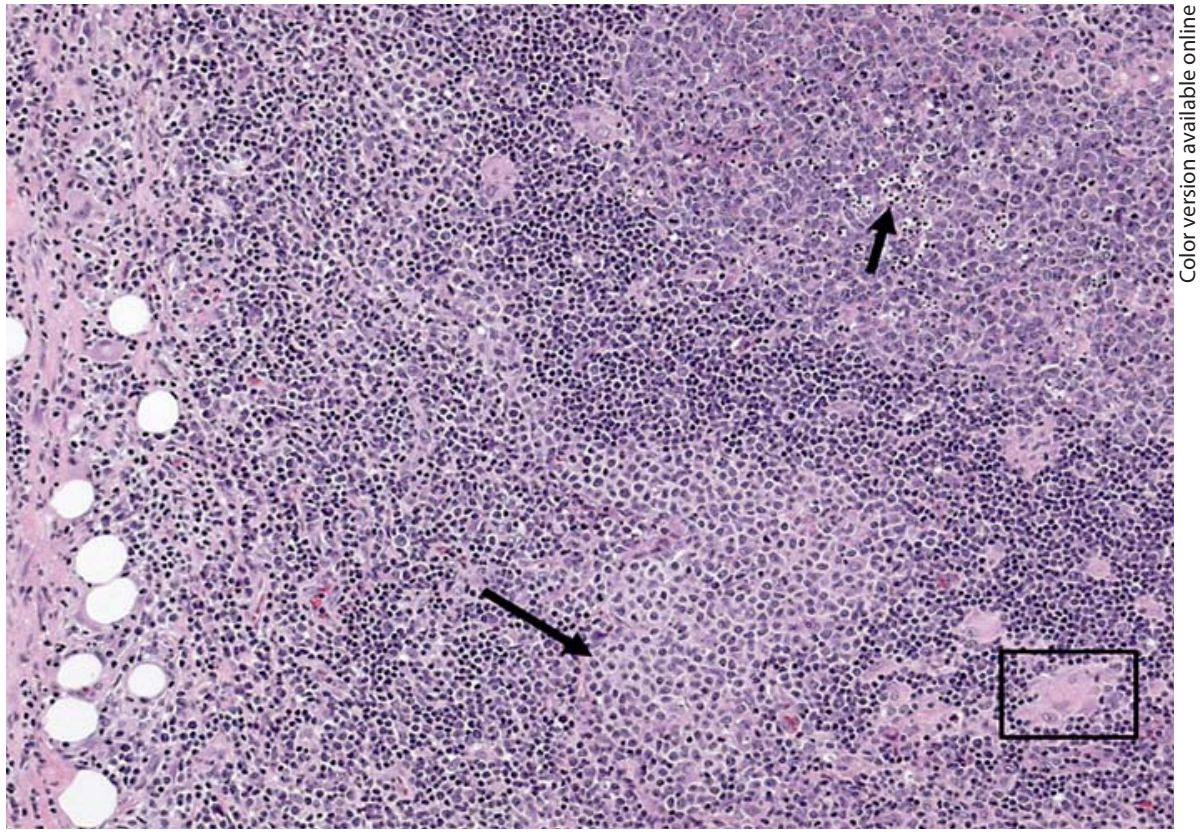

Ivanova/Glatz/Zippelius/Nicolas/Itin 
cleared (fig. 1C). The patient's cat has never had any symptoms of toxoplasmosis and the serologic test (IgM/IgG antibodies) as well as the PCR for oocyst in feces was negative at this time. Furthermore, no family member developed sings of acute toxoplasmosis. It is still unclear how our patient contracted this infection.

\section{Discussion}

We present an unusual case of acute toxoplasmosis which mimicked a malignant cervical lymphadenopathy in the draining area of a previously treated primary melanoma causing a diagnostic pitfall.

Toxoplasmosis is an infection with worldwide distribution which is caused by the intracellular protozoan parasite, T. gondii. The seroprevalence of $T$. gondii differs strongly in different parts of the Earth. In the USA, a seroprevalence of $9.0 \%$ has been reported during 1999-2004 in persons 1249 years of age [1]. In central Iran, an overall prevalence of $41.4 \%$ has been published [2] and $13.2 \%$ on Jeju Island, Korea, respectively [3]. In Europe, there is evidence that the burden of congenital toxoplasmosis is lower in Northern Europe than in the rest of the continent [4]. In cities, the prevalence of toxoplasmosis is lower than in non-metropolitan areas. It increases with age, but decreases with calendar time and varies according to geographical origin. In Switzerland, the seroprevalence for toxoplasmosis among pregnant women decreased steadily from 53\% (1982-1985) to 35\% (1999) [4]. Cats are the only animals in which T. gondii can complete its reproductive cycle. After ingestion of $T$. gondii, the parasite infects the gut epithelial cells of the cat, reproduces and is excreted as an infectious oocyst in the feces [5]. Humans acquire the infection from ingestion of oocyst-contaminated soil and water, from tissue cysts in undercooked meat, by transplantation, blood transfusion, laboratory accidents, or congenitally [1]. When non-cat species, including humans, ingest T. gondii oocysts, the organisms invade intestinal epithelium and disseminate throughout the body [5]. They encyst in any type of nucleated cell and can lie dormant within tissues for the life of the host. Jones et al. [6] reported that exposure to certain raw or undercooked foods and contact with kittens are risk factors for $T$. gondii infection. In immunocompetent hosts the acute T. gondii infection is asymptomatic. However, some patients may develop fever, mal- aise, chills, sweats, headaches or myalgias. Reviews reveal an incidence of cutaneous toxoplasmosis $<10 \%$ with a great variability of the skin lesions [7]. Vidal et al. [8] described a case of 16-year-old male patient after a stem cell transplantation which developed a subtle papular, erythematous rash erupted over his back and chest. The histology revealed an interface dermatitis with occasional necrotic keratinocytes, eligible for a graft-versus-host disease. However, $T$. gondii have been identified within the epidermal keratinocytes. Another case of toxoplasmosis occurred simultaneously with dermatomyositis has been seen in a 12 -yearold boy. Within 2 weeks after initiating therapy with sulfadiazine and pyrimethamine, dramatic clinical improvement has been noted [9]. A HIV-positive patient, presenting with hard and painful nodular lesions on the arms, hands and chest, had a negative serology for T. gondii, but the histopathologic examination of the lesion revealed foci of macrophages containing the protozoan parasite T. gondii [10]. In Italy, in 1980, during a period of 3 years Binazzi and Papini [11] observed 9 patients with evidence of trophozoites in skin lesions which presented different nonspecific cutaneous manifestations as ulcerative, purpuric teleangiectatic dermatosis of the lower limbs, relapsing erythema multiforme-like eruptions, erythema anulare centrifigum, lichenoid, lenticular or nummular patches as well as migrant, erysipelas-like edematous, cyanotic dermatitis. The most common manifestation of the acute toxoplasmosis is bilateral, symmetrical, nontender cervical adenopathy, which usually resolves, but in rare cases may persist chronically. The course is benign, self-limited and lasts from weeks to months. Toxoplasmosis has long been known as a major cause of perinatal morbidity. Acute infection in pregnancy may lead to fetal infection and subsequent fetal loss or birth of a manifestly or latently infected newborn with signs of hydrocephalus, cerebral calcification or debilitating ocular disease caused by chorioretinitis or anterior uveitis [12]. Toxoplasmosis encephalitis, pneumonitis, myocarditis/pericarditis, hepatitis, or polymyositis have been reported in patients with immunosuppression [1].

The diagnosis of toxoplasmosis is traditionally based on a combination of tissue biopsy and serological studies detecting antibodies against the parasite. However, if a patient's immune system is impaired, serologic tests may be falsely negative [13]. Viguer et al. [14] presented data about the cytological diagnosis of $T$. gondii, obtained with FNA biopsy which showed high sensitivity and specificity. A distinct histologic form of lymphadenitis observed in toxoplasmosis was described by PiringerKuchinka et al. [15] in 1958. At low magnification, there is periadenitis with capsular inflammation and scarring. Follicles are large and irregular with active germinal centers containing many tingible body macrophages. Scattered collections of pale staining parafollicular monocytoid B cells and small clusters of epithelioid histiocytes forming small granulomas are the most distinct features of toxoplasmic lymphadenitis [16]. The granulomas are smaller and less sharply defined than in sarcoidosis. Langhans giant cells or caseating necrosis is absent. Lymphadenitis in toxoplasmosis may be diagnosed in FNA cytology specimens if loosely aggregated epithelioid histiocytes and tingible body macrophages are present. The crescent-shaped organisms are only very rarely observed in aspirates except in severely immunocompromised patients.

Because of the difficulty of isolating the parasite, and the generally asymptomatic nature of the primary infection, the diagnosis of acquired toxoplasmosis in immunocompetent subjects is based on serologic tests: seroconversion to 2 isotypes (IgM and IgG antibodies) or a significant increase in the IgG titer plus IgM positivity [17]. Specific IgM is considered as an acute-phase marker. However, available assays have certain drawbacks, such as a risk of false-positive results due to antinuclear antibodies or rheumatoid factor or, owing to their high sensitivity detection of residual IgM, persistent months or even years after the primary infection [18]. Complementary techniques, including IgA detection and IgG avidity assay, can refine the date of infection. In a survey, Candolfi et al. [18] demonstrated that the IgG avidity test revealed a sensitivity of $100 \%$, a specificity of $92.7 \%$, a positive predictive value of $90 \%$, and a negative predictive value of $100 \%$. Pignanelli [19] demonstrated a high diagnostic accuracy of the real-time PCR which is an easy, accurate and rapid diagnostic technique in cases of borderline serologic tests.

The differential diagnosis of toxoplasmosis includes disorders presenting with disseminated lymphadenopathy: primary HIV infection, infectious mononucleosis, cat scratch disease and Hodgkin's lymphoma. Because the course is self-limiting and the $T$. gondii infection resolves spontaneously, immunocompetent and nonpreg- 
nant patients generally do not require treatment. If the symptoms are severe or prolonged beyond a few weeks the infection can be treated with the same therapy as in immunosuppressed patients.

Melanoma incidence rates continued to increase worldwide among white men (2.1\% per year) and white women (2.4\% per year) during 1999 through 2008. Rates increased for men aged over 55 years and women of all ages [20]. In the United States an estimated 68,130 new cases of malignant melanoma were predicted to be diagnosed in 2010, resulting in 8,700 deaths [21]. Approximately $20 \%$ of primary lesions are located on the head and neck, although this region accounts for only $9 \%$ of the total surface area of the body. Multiple factors contribute to this anatomic predilection, including higher levels of sun exposure and melanocyte content of the skin. Mortality rates among head and neck melanomas differ by site: lesions of the scalp and neck have the highest mortality, with a 10-year survival of $60 \%$. Tumors located on the ear, face, and eyelid have 10-year survival rates of 70, 80 and $90 \%$, respectively $[22,23]$. Advanced age, male sex, nodular morphological features, tumor thickness, ulceration, and Clark level V carry a significant risk of death for cutaneous head and neck melanoma, whereas facial melanomas have a favorable prognosis [23]. Occult lymph node metastasis is present in $15-20 \%$ of patients with melanoma of the head and neck and clinically negative nodes. According to Chai et al. [24], preoperative ultrasound (US) is not useful for identifying nodal metastasis in clinically node-negative melanoma patients undergoing sentinel node (SN) biopsy. In 471 patients, the overall sensitivity of US was $33.8 \%$, specificity $85.7 \%$, positive predictive value $36.5 \%$, and negative predictive value $84.2 \%$, respectively. The sensitivity and specificity appeared to improve somewhat with increasing Breslow depth. Sensitivity was highest for the neck, but specificity was highest for the groin [24]. However, the combination of preoperative US and US-guided FNA cytology can identify $65 \%$ of SN metastases and thus reduce the need for surgical SN procedures [25]. De Rosa et al. [22] reported a highly successful identification $(93.4 \%)$ of the $\mathrm{SN}$ in head and neck melanoma. However, despite the technical success, this procedure results in an elevated false-negative rate $(20.4 \%)$ compared with non-head and neck lesions.

In contrast to other skin cancers, melanoma is an aggressive neoplasm that may spread in an unpredictable manner to any organs of the body. This advocates the use of whole body imaging for tumor staging, in selected groups of patients. Disease dissemination may occur by direct extension from the primary site, via lymphatic spread to regional or distant lymph nodes, or by the hematogenous route. However, lymphatic spread to the regional draining area is the most common. Malignant tumors have a higher metabolic rate than normal tissues and generally overexpress numbers of specific membrane transporter proteins such as glucose transporters. Melanoma cells usually present with a high uptake of the radiolabeled glucose analog ${ }^{18} \mathrm{~F}$-FDG and can be visualized in vivo on PET imaging [26]. When coinvestigated with CT, accurate fusion of both metabolic and anatomic information enhances the staging performance of each individual technique. Indeed ${ }^{18} \mathrm{~F}$-FDG PET/CT scanning is more accurate in the detection of melanoma metastasis than CT, MRI, or ${ }^{18} \mathrm{~F}$-FDG PET alone [27-30]. In patients with advanced melanoma, ${ }^{18} \mathrm{~F}$-FDG PET/CT is at least as sensitive and definitely more specific than anatomic imaging modalities such as CT and MRI for the detection of metastatic disease $[28,29]$. Overall sensitivity and specificity of ${ }^{18} \mathrm{~F}$-FDG PET/CT in the staging of malignant melanoma are approximately 98\% [31]. However, ${ }^{18}$ F-FDG PET/CT lacks sensitivity for initial regional staging in stage I and IIA/B melanoma patients according to the American Joint Committee on Cancer 2009. Therefore, in primary invasive mel-anoma $\geq 1 \mathrm{~mm}$ in size, selective sentinel lymphadenectomy is considered as the standard of care approach in staging these patients. Moreover, ${ }^{18}$ F-FDG uptake is not tumor specific. As interpreting physicians, we need to be aware of these falsepositive and false-negative results. Technical causes of false-positive results may be due to misregistration artifact, injection artifact or imaging of metallic implants [31]. Sites of physiological ${ }^{18}$ F-FDG uptake include cerebral tissue, urinary system and liver, whereas sites of physiological ${ }^{18} \mathrm{~F}$ FDG activity include brown fat, adrenal activity, uterus and ovaries. An inflammation (surgery or radiotherapy, pelvic insufficiency fractures, granulomatous disease) can be also confused with malignancy [32-34]. Inflammatory cells such as neutrophils and activated macrophages at the site of infection or inflammation actively accumulate ${ }^{18} \mathrm{~F}-\mathrm{FDG}$. In the posttherapy setting, it has been reported that up to $40 \%$ of ${ }^{18} \mathrm{~F}$-FDG uptake occurs in nontumor tissue. Infection is one of the most common causes of false-positive ${ }^{18} \mathrm{~F}$-FDG PET/CT findings after chemotherapy. These patients are susceptible to a wide variety of infections, including upper respiratory chest infections, pneumonia, colitis, cholecystitis and cellulitis. Reactivation of tuberculous infection can occur in immunocompromised patients postche-motherapy, and correlation with CT imaging can prevent misdiagnosis in suspected cases. Atypical fungal and parasitic infections such as cryptococcosis, pneumocystis and paragonimiasis can also present as false-positives on ${ }^{18} \mathrm{~F}$-FDG imaging [32]. Long and Smith [32] presented the case of a 67-year-old male for restaging of a laryngeal carcinoma with increased ${ }^{18} \mathrm{~F}$ FDG uptake in the left lower neck and left mediastinum. CT demonstrated lymphadenopathy in these areas. Biopsy of the left lower neck node confirmed sarcoidosis. In female patients, acute and chronic inflammation, physiologic lactation, and benign breast masses, including silicone granuloma, fat necrosis, fibroadenoma, and postsurgical changes can mimic breast cancer on ${ }^{18}$ F-FDG PET and PET/CT [35]. Falsenegative results have been reported with marrow stimulation and in nodes adjacent to brown fat uptake.

\section{Conclusion}

Acute toxoplasmosis can mimic malignant lymphadenitis. Serologic tests and a highly specific histologic pattern provide the diagnosis of $T$. gondii infection which runs a benign self-limited course in immunocompetent patients. ${ }^{18} \mathrm{~F}-\mathrm{FDG} \mathrm{PET} / \mathrm{CT}$ shows very high sensitivity and specificity in staging of advanced malignant melanoma, but lacks sensitivity for initial regional staging in stage I and IIA/B melanoma patients according to the American Joint Committee on Cancer 2009. However, ${ }^{18} \mathrm{~F}$ FDG uptake is not tumor specific. Therefore, different pathologic and physiologic conditions as inflammation, infection, surgery or artifact may cause false-positive results.

\section{Disclosure Statement}

The authors have no conflicts of interest to declare. 


\section{References}

-1 Elmore SA, Jones JL, Conrad PA, Patton S, Lindsay DS, Dubey JP: Toxoplasma gondii: epidemiology, feline clinical aspects, and prevention. Trends Parasitol 2010;26:190-196.

-2 Mostafavi SN, Ataei B, Nokhodian Z, Yaran M, Babak A: Seroepidemiology of Toxoplasma gondii infection in Isfahan province, central Iran: a population based study. J Res Med Sci 2011;16:496-501.

-3 Hong SJ, Chong CK, Lee K, Kim TS, Hong YP, Ahn HJ, Kim HY, Ko AR, Kim YJ, Nam HW: Maintained seroprevalence of toxoplasmosis among the residents of Jeju Island, Korea. Korean J Parasitol 2011;49:309-311.

-4 Boubaker K, Raeber PA, Vaudaux B, Bucher HC, Garweg JG, Hoesli I, Kind C, Hohlfeld P: Toxoplasmosis during pregnancy and infancy. A new approach for Switzerland. Swiss Med Wkly 2008;138(suppl 168):1-8.

-5 Tenter AM, Heckeroth AR, Weiss LM: Toxoplasma gondii: from animals to humans. Int $\mathrm{J}$ Parasitol 2000;30:1217-1258.

-6 Jones JL, Dargelas V, Roberts J, Press C, Remington JS, Montoya JG: Risk factors for Toxoplasma gondii infection in the United States. Clin Infect Dis 2009;49:878-884.

-7 Mawhorter SD, Effron D, Blinkhorn R, Spagnuolo PJ: Cutaneous manifestations of toxoplasmosis. Clin Infect Dis 1992;14:10841088.

8 Vidal CI, Pollack M, Uliasz A, del Toro G, Emanuel PO: Cutaneous toxoplasmosis histologically mimicking graft-versus-host disease. Am J Dermatopathol 2008;30:492-493.

$\checkmark 9$ Pollock JL: Toxoplasmosis appearing to be dermatomyositis. Arch Dermatol 1979;115: 736-737.

10 Fong MY, Wong KT, Rohela M, Tan LH, Adeeba K, Lee YY, Lau YL: Unusual manifestation of cutaneous toxoplasmosis in a HIV positive patient. Trop Biomed 2010;27. 447-450.

11 Binazzi M, Papini M: Cutaneous toxoplasmosis. Int J Dermatol 1980;19:332-335.

12 Commodaro AG, Belfort RN, Rizzo LV, Muccioli C, Silveira C, Burnier MN Jr, Belfort R Jr: Ocular toxoplasmosis: an update and review of the literature. Mem Inst Oswaldo Cruz 2009;104:345-350.

13 Chen X, Remotti F, Tong GX, Gorczyca E, Hamele-Bena D: Fine-needle aspiration cytology of subcutaneous toxoplasmosis: a case report. Diagn Cytopathol 2010;38:716-720.
14 Viguer JM, Jimenez-Heffernan JA, LopezFerrer P, Conzalez-Peramato, P, Vicandi B: Fine needle aspiration of toxoplasmic (Piringer-Kuchinka) lymphadenitis: a cytohistologic correlation study. Acta Cytol 2005;49:139143.

15 Piringer-Kuchinka A, Martin I, Thalhammer O: Superior cervicalnuchal lymphadenitis with small groups of epitheloid cell proliferation. Virchows Arch 1958;331:522-535.

16 Yildirim AC, Bostanci H, Yilmaz ED, Kutluer $\mathrm{N}$, Kargici H: Toxoplasma lymphadenitis mimicking malign axillary lymphadenopathy of a left breast mass. Updates Surg 2012.

17 Silva-Dos-Santos PP, Barros GB, Mineo JR, Silva DA, Menegaz MH, Serufo JC, Dietze R, Martins-Filho OD, Lemos EM: Flow cytometry-based algorithm to analyze the anti-fixed Toxoplasma gondii tachyzoites IgM and IgG reactivity and diagnose human acute toxoplasmosis. J Immunol Methods 2012;378:3343.

18 Candolfi E, Pastor R, Huber R, Filisetti D, Villard O: IgG avidity assay firms up the diagnosis of acute toxoplasmosis on the first serum sample in immunocompetent pregnant women. Diagn Microbiol Infect Dis 2007;58:8388.

19 Pignanelli S: Laboratory diagnosis of Toxoplasma gondii infection with direct and indirect diagnostic techniques. Indian J Pathol Microbiol 2011;54:786-789.

20 Simard EP, Ward EM, Siegel R, Jemal A: Cancers with increasing incidence trends in the United States: 1999 through 2008. CA Cancer J Clin 2012;62:118-128.

21 Jemal A, Siegel R, Xu J, Ward E: Cancer statistics, 2010. CA Cancer J Clin 2010;60:277300.

22 De Rosa N, Lyman GH, Silbermins D, Valsecchi ME, Pruitt SK, Tyler DM, Lee WT: Sentinel node biopsy for head and neck melanoma: a systematic review. Otolaryngol Head Neck Surg 2011;145:375-382.

23 Golger A, Young DS, Ghazarian D, Neligan PC: Epidemiological features and prognostic factors of cutaneous head and neck melanoma: a population-based study. Arch Otolaryngol Head Neck Surg 2007;133:442-447.

24 Chai CY, Zager JS, Szabunio MM, Marzban SS, Chau A, Rossi RM, Sondak VK: Preoperative ultrasound is not useful for identifying nodal metastasis in melanoma patients undergoing sentinel node biopsy: preoperative ultrasound in clinically node-negative melanoma. Ann Surg Oncol 2012;19:1100-1106.

25 Voit C, Van Akkooi AC, Schäfer-Hesterberg G, Schoengen A, Kowalczyk K, Roewert JC, Sterry W, Eggermont AM: Ultrasound morphology criteria predict metastatic disease of the sentinel nodes in patients with melanoma. J Clin Oncol 2010;28:847-852.
26 Gillies RJ, Robey I, Gatenby RA: Causes and consequences of increased glucose metabolism of cancers. J Nucl Med 2008;49(suppl 2):24S-42S

27 Reinhardt MJ, Joe AY, Jaeger U, Huber A, Matthies A, Bucerius J, Roedel R, Strunk H, Bieber T, Biersack HJ, Tüting T: Diagnostic performance of whole body dual modality ${ }^{18} \mathrm{~F}$-FDG PET/CT imaging for $\mathrm{N}$ - and M-staging of malignant melanoma: experience with 250 consecutive patients. J Clin Oncol 2006; 24:1178-1187.

28 Mottaghy FM, Sunderkötter C, Schubert R, et al: Direct comparison of $\left.{ }^{18}{ }^{18} \mathrm{~F}\right] \mathrm{FDG}$ PET/CT with PET alone and with side-by-side PET and CT in patients with malignant melanoma. Eur J Nucl Med Mol Imaging 2007;34:13551364.

29 Strobel K, Dummer R, Husarik DB, et al High-risk melanoma: accuracy of FDG PET/ CT with added CT morphologic information for detection of metastases. Radiology 2007; 244:566-574

30 Pfannenberg C, Aschoff P, Schanz S, et al: Prospective comparison of $18 \mathrm{~F}$-fluorodeoxyglucose positron emission tomography/computed tomography and whole-body magnetic resonance imaging in staging of advanced malignant melanoma. Eur J Cancer 2007;43: 557-564.

31 Essler M, Link A, Belloni B, Mirceva V, Souvatzoglou $M$, Thaler $M$, Haller $B$, Hein $R$, Krause BJ: Prognostic value of $\left[{ }^{18} \mathrm{~F}\right]$-fluorodeoxy-glucose PET/CT, S100 or MIA for assessment of cancer-associated mortality in patients with high risk melanoma. PLoS One 2011;6:e24632.

32 Long NM, Smith CS: Causes and imaging features of false positives and false negatives on F-PET/CT in oncologic imaging. Insights Imaging 2011;2:679-698.

33 Ozgiray E, Oner K, Ovul I: HIV related toxoplasmic encephalitis mimicking multiple metastasis: case report. Turk Neurosurg 2007;17: 207-210.

34 Spieth K, Risse J, Kaufmann R, Gille J: Challenging cases and diagnostic dilemmas: case 3 . Positron emission tomography scan mimicking lymph node metastases in a high-risk melanoma patient. J Clin Oncol 2002;20:33493351.

- 35 Adejolu M, Huo L, Rohren E, Santiago L, Yang WT: False-positive lesions mimicking breast cancer on FDG PET and PET/CT. AJR Am J Roentgenol 2012;198:W304-W314. 